\title{
Les stratégies des « marques nationales » face aux « marques de distributeurs »
}

\author{
Catherine Parissier et Julie Laurence \\ Université de Sherbrooke
}

$\int$ es marques de distributeurs (MDD), nommées également «marques privées », « marques maison» ou encore "marques de détaillants », connaissent depuis quelques années au Canada comme en Europe un essor considérable et elles représentent désormais une part conséquente des ventes de produits au détail dans les pays où elles sont implantées. Ainsi, les ventes de MDD au Québec et au Canada atteignent maintenant respectivement $18,7 \%$ et $25,1 \%$ de parts de marché sur les ventes au détail en volume, et au Québec les ventes de ces produits s'accroissent depuis 2001 de $15 \%$ par an ${ }^{1}$.

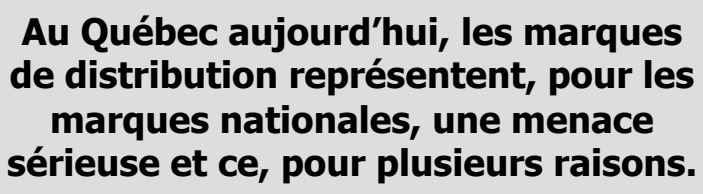

marge de profit sur le prix de vente consommateur de $15 \%$ supérieure à celle des marques nationales ${ }^{4}$. Les distributeurs ont donc un intérêt financier certain à promouvoir ces produits sur les tablettes de leurs magasins. De plus, la concentration de la distribution étant très élevée au Québec (les trois principaux détaillants de supermarchés accaparent environ $70 \%$ du marché québécois du commerce de détail), les effets de ces aménagements de gamme des détaillants sur les ventes des marques nationales peuvent être considérables. Ce danger est d'autant plus menaçant que la grande majorité des Québécois achètent et apprécient ces MDD : $75 \%$ des Québécois les achètent (dont $41 \%$ régulièrement et $34 \%$ occasionnellement) et $82 \%$ les jugent de qualité égale ou supérieure aux marques nationales disponibles 5 .

La menace représentée par les MDD peut engendrer pour les marques nationales des pertes de parts de marché, une érosion du profit ou encore une dilution du capital de marque. Les gestionnaires de marque sont donc dans l'obligation de concevoir des stratégies pour agir face à cette nouvelle réalité concurrentielle. Plusieurs travaux académiques portant sur ce sujet ont été menés en Europe et aux Etats-Unis, mais peu l'ont été dans le contexte canadien et québécois. Le but de cette recherche est donc de recenser les stratégies appliquées au Québec par les marques nationales face à la menace des MDD, d'en identifier l'importance relative mais aussi d'étudier si certains facteurs (la catégorie de produit ou la position de la marque dans sa catégorie) peuvent influencer les gestionnaires de marque dans leur décision de choix et de mise en œuvre de ces stratégies.

Dans une première partie, une synthèse de la littérature sera exposée sur les stratégies généralement 
adoptées par les marques nationales vis-à-vis des MDD, suivie en seconde partie par une présentation de nos questions de recherche et de la méthodologie adoptée pour recueillir des données auprès de gestionnaires de marques québécois. Dans une troisième partie, nous présenterons les principaux résultats de nos investigations pour conclure sur les implications managériales pour les gestionnaires de marques au Québec et sur les pistes de recherche futures.

\section{Les stratégies des marques nationales face aux marques de distributeurs}

Plusieurs auteurs présents dans la littérature scientifique marketing ${ }^{6}$ concluent qu'il existe quatre stratégies principales qu'un gestionnaire de marque peut employer afin de pérenniser sa marque face au développement des MDD : 1) attendre et ne rien faire, 2) accroître la distance par rapport aux MDD par la qualité, l'innovation et la variété, 3) introduire une marque "bas de gamme" et 4) accepter de fabriquer une MDD. Nous expliciterons successivement chacune de ces stratégies.

\section{L'accroissement de la distance entre la marque nationale et les MDD par une politique produit peut être obtenu en offrant soit un meilleur rapport qualité-prix, soit une innovation significative, ou encore en proposant une plus grande variété de choix de produits associés à la marque.}

\section{Attendre et ne poser aucune action}

Le gestionnaire de marque optant pour cette stratégie se place en position d'observation et d'attente vis-àvis des MDD et élabore sa stratégie essentiellement par rapport aux autres marques nationales, comme si les MDD ne s'apparentaient pas à sa concurrence directe. L'avantage consiste à reporter sur d'autres concurrents l'initiative de contrer les MDD et à leur faire supporter les coûts liés à cette politique (par exemple, le coût d'introduction d'une innovation sur le marché pour contrer une copie de la part d'une MDD, ou encore le coût lié à la suppression de produits par le distributeur en conséquence de l'action menée contre les produits de sa «marque maison »). Cette stratégie peut toutefois également avoir des conséquences né- fastes pour la marque nationale telles que la perte progressive de parts de marché face aux MDD et la détérioration de l'image de la marque auprès des consommateurs qui, en l'absence de réaction visible de la marque nationale, ne perçoivent plus de valeur ajoutée par rapport à la MDD. Des travaux menés en Europe (Pays-Bas) démontrent que cette stratégie est encore employée par certains gestionnaires de marque.

\section{Accroître la distance de la marque nationale par rapport à la MDD}

Cette stratégie peut se construire en améliorant soit le produit en tant que tel, soit le capital de la marque afin de différencier cette dernière de ses compétiteurs, ces deux voies n'étant pas mutuellement exclusives. L'accroissement de la distance entre la marque nationale et les MDD par une politique produit peut être obtenu en offrant soit un meilleur rapport qualité-prix (c'est-àdire en améliorant le produit existant tout en maintenant son prix de vente), soit une innovation significative, ou encore en proposant une plus grande variété de choix de produits associés à la marque. L'accentuation de la distance par le capital de marque peut quant elle s'obtenir par des campagnes agressives de publicité ou de communication en général (par exemple, en conjuguant promotion des ventes et publicité à grande échelle). Cette stratégie mobilise des ressources importantes et elle est fréquemment utilisée par des marques qui jouissent dans leur marché d'une position de leader.

\section{Introduire une marque « bas de gamme »}

La troisième stratégie consiste à introduire sur le marché une marque «bas de gamme » (généralement sans lien apparent avec la marque nationale concernée), appelée aussi «value flanker » dans la littérature anglo-saxonne, afin de limiter les effets de la MDD « sans nom » sur la clientèle recherchant essentiellement des produits à bas prix. Cette approche donne la possibilité à la marque nationale de préserver son image, car la marque « bas de gamme » qu'elle introduit pour concurrencer la MDD semble sans lien direct avec elle aux yeux des consommateurs. Elle a également comme avantage d'éviter les guerres de prix ou les surenchères promotionnelles entre la MDD et la marque nationale et d'augmenter au final les parts de marché du fabricant. Ce dernier peut ainsi exploiter une éventuelle capacité de production excédentaire et accéder ainsi à des gains supplémentaires qui n'au- 
raient pu être obtenus autrement. La marque nationale peut également s'assurer de conserver une place intéressante sur les tablettes puisque son exposition est la somme de sa présence en tant que marque nationale et comme marque de premier prix. Cette stratégie offre toutefois certains inconvénients. Une marque «bas de gamme » peut, en effet, cannibaliser les ventes de la marque nationale, et même si l'effort marketing pour supporter la marque est mineur, elle détourne des marques principales de l'entreprise une part du budget marketing. Par ailleurs, cette stratégie peut miner les relations du fabricant avec ses distributeurs, car la marque «bas de gamme » concurrence de façon évidente les MDD des détaillants, alors que la tendance de fond de ce secteur est plus à la collaboration entre distributeurs et détaillants qu'à la confrontation. Quelques marques adoptent cette stratégie afin d'offrir des niveaux distincts de qualité sur le marché.

\section{Accepter de fabriquer une MDD}

La quatrième stratégie qui consiste à accepter de fabriquer une MDD pour un détaillant en particulier comporte deux options : fabriquer une marque «bas de gamme » pour le détaillant ou, au contraire, une marque «haut de gamme» (par exemple, pour le détaillant Loblaw au Canada, le fabricant a la possibilité de fabriquer soit une marque «Sans Nom », soit une marque "Choix du Président»). Cette stratégie présente trois avantages majeurs que nous exposerons successivement. Le premier est économique; en effet, accepter de fabriquer une MDD permet au fabricant de mettre sur le marché ses excédents de production et de réaliser ainsi des profits additionnels. Le second repose sur la construction d'une meilleure relation d'affaires avec le distributeur; plutôt que de s'engager dans une guerre des prix qui affaiblit les deux joueurs, il semble préférable pour le fabricant de se diriger vers une coopération qui donne la possibilité à la marque de se différencier tout en acceptant de créer une MDD pour son partenaire distributeur. Enfin, le troisième avantage est celui de la position concurrentielle que le fabricant peut prétendre améliorer. Accepter de fabriquer pour un distributeur une MDD offre au fabricant une place assurée et favorable sur les tablettes, ce qui signifie par le fait même un meilleur potentiel de ventes et donc un accroissement de parts de marché, ou encore la possibilité d'évincer un concurrent. De plus, le fait de fabriquer une MDD peut permettre à l'entreprise de protéger à terme une innovation qu'elle accepte de concéder au distributeur (ce dernier peut en effet bloquer l'accès de ses tablettes à des copies potentielles pour protéger sa propre MDD).

\section{Questions de recherche et méthodologie adoptée}

\section{Questions de recherche}

La revue de littérature nous a permis d'identifier quatre stratégies principales permettant aux marques nationales de contrer la menace des MDD. L'absence d'écrits sur les manœuvres des gestionnaires de marques nationales au Québec faisant pourtant face à une situation similaire nous laisse percevoir la nécessité d'aller plus en profondeur sur ce sujet. Notre objectif est donc de dresser un portrait de la situation au Québec et de conclure sur la formulation de recommandations. Advenant la possibilité qu'une cinquième stratégie puisse être observée, nous avons donc posé notre cadre conceptuel comme présenté à la figure 1 (page suivante). Notre question de recherche principale (Q1) a donc été formulée comme suit : Existe-t-il seulement cinq stratégies possibles de gestion des marques nationales au Québec face aux MDD, soit (1) attendre et ne poser aucune action, (2) accroître la distance par rapport aux MDD, (3) introduire une marque bas de gamme, (4) accepter de fabriquer une MDD ou (5) toute autre stratégie non encore identifiée? Dans un second temps, après les avoir identifiées, nous nous intéresserons à l'importance relative de chacune de ces cinq stratégies auprès des gestionnaires de marques nationales au Québec.

\section{Le fait de fabriquer une MDD peut permettre à l'entreprise de protéger à terme une innovation qu'elle accepte de concéder au distributeur.}

Certaines variables peuvent toutefois avoir une influence sur les décisions stratégiques des gestionnaires de marque et modérer les relations entre les variables de notre modèle. Ainsi, la catégorie de produits (ensemble de produits complémentaires et substituables pour répondre à un besoin des consommateurs) pourrait être considérée comme déterminante dans les choix stratégiques vis-à-vis des $\mathrm{MDD}^{7}$. Les MDD seraient particulièrement performantes dans des catégories fortement concentrées (où le nombre de marques disponibles est peu élevé), car la différenciation entre 
les marques serait plus ardue; dans des catégories de produits offrant peu de variété (où le nombre de saveurs ou d'options disponibles dans la catégorie est faible), les MDD domineraient plus aisément le marché, nécessitant une réaction stratégique plus vive de la part des marques nationales. Nous estimons donc nécessaire de poser sur cette première variable modératrice de notre modèle la question de recherche suivante (Q2) : La catégorie de produits, selon sa concentration et sa variété, modère-t-elle l'importance relative de chacune des cinq stratégies principales dans l'élaboration des stratégies globales des marques nationales au Québec face aux MDD?

La position d'une marque dans le marché peut s'illustrer par l'une des quatre situations suivantes : le « leader » occupe la position dominante sur le marché, le « challenger » est son principal rival avec une posi- tion de second, le «suiveur» fait partie des autres joueurs du marché, alors que le "spécialiste» est un acteur qui se situe dans une niche on un créneau bien spécifique du marché. La position de la marque nationale peut influencer fortement les décisions des gestionnaires puisque plusieurs MDD « haut de gamme » semblent choisir de poser des actions similaires aux marques nationales leaders de leur marché (Sayman et al.). Ainsi, il nous semble essentiel de considérer l'influence de la position de la marque sur notre modèle conceptuel et de poser la question de recherche suivante $(\mathrm{Q} 3)$ : La position de la marque nationale dans la catégorie modère-t-elle l'importance relative de chacune des cinq stratégies principales dans l'élaboration des stratégies globales des marques nationales au Québec face aux MDD ? Le modèle intégrant ces deux variables modératrices est présenté de façon globale à la figure 1 .

Figure 1 - Modèle conceptuel de la recherche

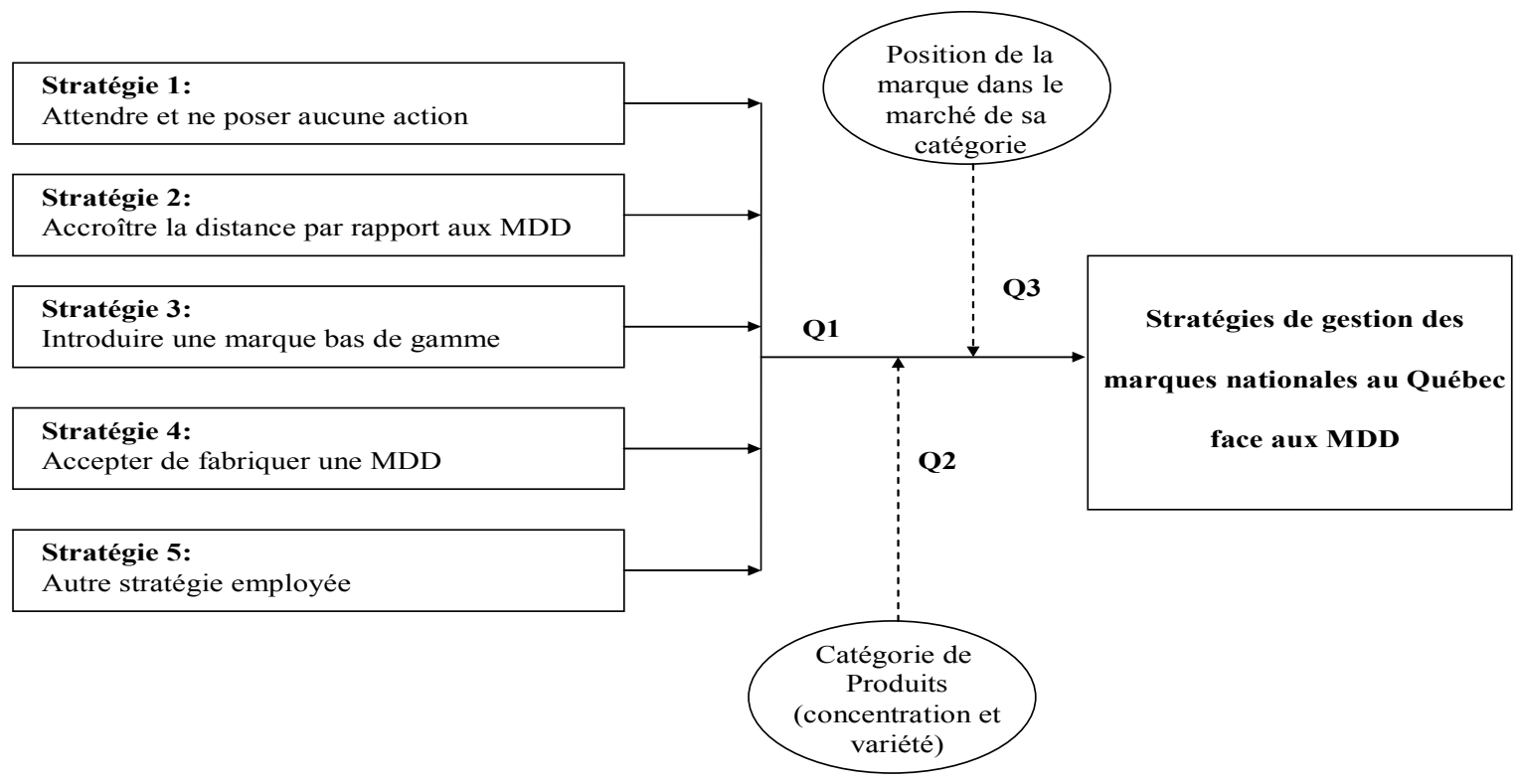

\section{Méthodologie}

L'étude réalisée étant essentiellement exploratoire, une méthode qualitative de collecte de données a été retenue pour notre recherche.

Les marques canadiennes agroalimentaires gérées au Québec et disponibles dans les principales épiceries québécoises ont constitué les «individus» de la population de l'étude (l'importance de l'industrie des aliments et des boissons au niveau fédéral et provin- cial a essentiellement motivé le choix de ce secteur ${ }^{8}$ ). Une base de données composée de 419 marques alimentaires canadiennes présentes en magasins d'alimentation au Québec, réparties en 22 catégories permettant une représentation de toutes les marques offertes dans les trois principales bannières d'épicerie régulières au Québec ${ }^{9}$ (IGA-Sobey's, Métro et Provigo-Loblaw), a été utilisée afin de constituer l'échantillon final ${ }^{10}$ ainsi bâti pour respecter la diversité des catégories de produits, la concentration des marques et des MDD dans chaque catégorie et enfin les quatre 
positions concurrentielles possibles des marques sur leur marché (tableau 1). Les gestionnaires de marques nationales œuvrant au Québec ont constitué les unités d'échantillonnage interrogées : 82 ont ainsi été contactées par téléphone et 21 ont finalement accepté de participer à l'enquête (11 en grande entreprise et 10 en PME).

La technique de cueillette des données par entrevues individuelles semi-dirigées a été retenue en raison du caractère confidentiel du sujet abordé et du profil des interviewés plus faciles à rejoindre sur leur lieu de travail.

Les entrevues ont été réalisées de mai à juillet 2004, en « face à face », sur la base d'un guide d'entretien, et elles ont fait l'objet d'un enregistrement « audio » afin d'en faciliter l'exploitation. Les entrevues ont été retranscrites sous forme de verbatim, puis étudiées selon le principe de l'analyse thématique.

\section{Tableau 1 - Profil de l'échantillon final de la recherche ${ }^{11}$}

\begin{tabular}{|c|c|c|c|c|c|c|c|c|}
\hline \multicolumn{9}{|c|}{ Profil de l'échantillon final selon la catégorie de produit } \\
\hline Aliments réf & & 4,8 & \multicolumn{2}{|c|}{$\begin{array}{l}\text { Jus, boissons et nectars } \\
\text { en conserve }\end{array}$} & 9,5 & \multicolumn{2}{|c|}{ Boissons alcoolisées } & 4,8 \\
\hline $\begin{array}{l}\text { Boissons gaz } \\
\text { eaux embout }\end{array}$ & & 4,8 & \multicolumn{2}{|c|}{ Produits à grignoter } & 9,5 & \multicolumn{2}{|c|}{ Charcuterie } & 4,8 \\
\hline Bases pour $b$ & & 4,8 & \multirow{2}{*}{\multicolumn{2}{|c|}{ Légumes en conserve }} & 4,8 & \multirow{2}{*}{\multicolumn{2}{|c|}{ Produits laitiers }} & 33,3 \\
\hline Produits de & ngerie & 19,0 & & & & & & \\
\hline \multicolumn{9}{|c|}{ Profil de l'échantillon final selon la concentration des MDD dans la catégorie } \\
\hline Très élevée & 19,0 & \multicolumn{2}{|c|}{ Élevée $\quad 1$} & Moyenne & & 28,6 & Faible & 38,1 \\
\hline \multicolumn{9}{|c|}{ Profil de l'échantillon final selon la concentration des marques dans la catégorie } \\
\hline Très élevée & 47,6 & \multicolumn{2}{|c|}{ Élevée $\quad 9,5$} & Moyenne & & 38,1 & Faible & 4,8 \\
\hline \multicolumn{9}{|c|}{ Profil de l'échantillon final selon la position de la marque dans la catégorie } \\
\hline Leader & 66,7 & \multicolumn{2}{|c|}{ Challenger } & Suiveur & & 9,5 & Spécialiste & 0 \\
\hline
\end{tabular}

\section{Les principaux résultats de la recherche}

Les 21 gestionnaires rencontrés ont tous indiqué qu'ils utilisaient, face aux MDD, une des cinq stratégies pressenties, ce qui nous permet de conclure que la première question de recherche de notre modèle conceptuel est validée (tableau 2). Les deux stratégies les plus utilisées sont, premièrement, la stratégie 2 qui vise à accroître la distance vis-à-vis des MDD, puis deuxièmement, la stratégie 4 qui prône la fabrication d'une ou plusieurs MDD pour les détaillants. Ces deux stratégies seront détaillées ci-dessous.

Les MDD offrent de plus en plus de produits de qualité et menacent la position des marques nationales. Les fabricants travaillent donc sur l'innovation (extensions, formats améliorés ou recettes innovatrices) dans le but de promouvoir des caractéristiques origi- nales pour leurs produits et, de ce fait, une valeur perçue supérieure aux MDD pour les consommateurs. Ils tentent également de véhiculer leur expertise et leur savoir-faire afin de différencier leurs marques et de renforcer leur positionnement original. Ils indiquent que cette stratégie leur permet de fidéliser leur clientèle, de distancer leurs concurrents en parts de marché, mais aussi de susciter l'intérêt des distributeurs qui les approchent pour fabriquer leurs propres marques. Ainsi, la seconde stratégie citée consiste à accepter de fabriquer une MDD. Trois motivations principales sont évoquées pour justifier cette option : économique (couvrir les frais fixes ou écouler une production excédentaire), concurrentielle (accroître les parts de marché et obtenir un meilleur emplacement sur les tablettes) et relationnelle (conserver la marque nationale en magasin et développer des alliances avec les distributeurs, par exemple à l'exportation). Cette se- 
conde stratégie est reconnue comme très profitable, car la marque ainsi créée dessert un segment «protégé » par les distributeurs qui y puisent eux aussi une source importante de rentabilité. Toutefois, elle doit être encadrée contractuellement et les gestionnaires suggèrent de ne pas aller dans le sens des MDD «haut de gamme» qui cannibalisent leurs propres ventes et minent leur image d'expertise.
D'autres stratégies (stratégie 5) ont également été évoquées, soit se rapprocher des MDD (dans les situations où la MDD devient leader de sa catégorie ou encore lorsque la catégorie recèle peu d'innovation, ce qui engendre peu de fidélité des consommateurs), ou encore introduire une marque " haut de gamme » pour conquérir un terrain où les MDD sont encore pas ou fort peu présentes.

\section{Tableau 2 - Fréquence de citation des stratégies utilisées par les gestionnaires de marques au Québec face aux MDD}

\begin{tabular}{lccc}
\multicolumn{1}{c}{ Stratégies } & Principale & Secondaire & Total \\
\hline Stratégie 1 : Attendre et ne poser aucune action & 2 & 0 & 2 \\
Stratégie 2 : Accroître la distance par rapport aux MDD & 14 & 4 & $\mathbf{1 8}$ \\
Stratégie 3 : Introduire une marque « bas de gamme » & 2 & 0 & 2 \\
Stratégie 4 : Accepter de fabriquer une MDD & 2 & 9 & $\mathbf{1 1}$ \\
Stratégie 5 : Autre (se rapprocher des MDD) & 1 & 0 & 1 \\
\hline Total (nombre de citations) & $\mathbf{2 1}$ & $\mathbf{1 3}$ & $\mathbf{3 4}$
\end{tabular}

Ces résultats nous ont donc également montré que les gestionnaires utilisaient fréquemment des combinaisons des cinq stratégies fondamentales exposées dans la recherche. L'analyse de ces combinaisons (tableau 3) montre que la stratégie combinée prioritaire est celle qui vise à la fois l'accroissement de distance visà-vis des MDD et la fabrication de marques privées. Un des gestionnaires de marques explique en effet: « [C'est une] bonne stratégie, car ça donne du volume et ça amène une bonne stabilité au niveaux des opérations [et] de la production... Je serais prêt à en faire plus, car ça permet aussi de donner un meilleur espace en tablette pour ta maque nationale ». Toutefois ce choix est essentiellement le fait des grandes entreprises (60\% des citations) qui possèdent des marques fortes appuyées par des moyens financiers conséquents.

Les résultats montrent également que la catégorie de produit et la position de la marque dans sa catégorie modèrent le choix des stratégies possibles ou de leur combinaison (questions de recherche 2 et 3 ).

\section{Tableau 3 - Fréquence de citation des stratégies combinées utilisées par les gestionnaires de marques au Québec face aux MDD}

\begin{tabular}{llc}
\multicolumn{1}{c}{ Stratégie prioritaire } & \multicolumn{1}{c}{ Stratégie secondaire } & Fréquence \\
\hline Accroître la distance / MDD (strat. 2) & $\begin{array}{l}\text { Accepter de fabriquer une MDD } \\
\text { (strat. 4) }\end{array}$ & 82 \\
Introduire une marque « bas de gamme » (strat. 3) & $\begin{array}{l}\text { Accroître la distance vis-à-vis des } \\
\text { MDD (strat. 2) }\end{array}$ & 2 \\
Se rapprocher des MDD (strat. 5) & $\begin{array}{l}\text { Accepter de fabriquer une MDD } \\
\text { (strat. 4) }\end{array}$ & 15 \\
Accepter de fabriquer une MDD (strat. 4) & $\begin{array}{l}\text { Accroître la distance vis-à-vis des } \\
\text { MDD (strat. 2) }\end{array}$ & 2 \\
\hline Total (nombre de citations) & & 15 \\
\hline
\end{tabular}


Les catégories offrant peu de variété sont très attaquées par les MDD, car les consommateurs recherchent le meilleur prix possible pour ces produits qu'ils considèrent comme courants. Les MDD essaient toutefois d'investir de plus en plus les catégories à forte variété, notamment par leurs produits « haut de gamme» et, de ce fait, les stratégies des marques nationales doivent s'adapter, car ces catégories deviennent très concurrentielles. Ainsi, un gestionnaire précise : «Il faut augmenter le support, varier l'offre si une marque haut de gamme privée arrive. Plus c'est concurrentiel, plus les stratégies évoluent en fonction de cela ».

Les gestionnaires ont également clairement indiqué que la position de leur marque influence grandement les stratégies à prendre. Une marque leader donne le ton en ce qui concerne les innovations, les promotions, les prix et la distribution, et cette position faciliterait les relations avec les détaillants. La marque leader attire les clients en magasin et les fidélise et, fréquemment, la marque nationale leader est celle qui peut le plus aisément répondre favorablement et qualitativement à la demande exprimée par le détaillant de lancer sur le marché sa propre marque privée. De surcroît, la majorité des gestionnaires ayant adopté une autre stratégie que celle qui consiste à accroître la distance vis-à-vis des MDD (stratégie 2) se situent actuellement en position de challenger ou de suiveur.

\section{Conclusion (implications managériales et avenues de recherche)}

Cinq stratégies principales ont donc été recensées auprès des gestionnaires de marques québécois face à l'émergence des MDD. La majorité travaillent à l'accroissement de la distance de leurs marques vis-à-vis des MDD par l'action conjuguée de l'innovation et du renforcement de leur image (stratégie 2) et/ou acceptent de fabriquer une marque privée pour un distributeur (stratégie 4). On note toutefois une évolution de la perception de cette dernière option. Elle est vue comme incontournable (il est très difficile aujourd'hui pour un fabricant de refuser de s'engager dans cette voie), voire obligatoire bien que profitable, mais elle peut placer les gestionnaires de marques dans une situation précaire (dépendance, cannibalisation des ventes de la marque nationale). La stratégie d'attente (stratégie 1) est encore citée mais apparaît de moins en moins réaliste auprès de consommateurs qui re- cherchent toujours plus de valeur ajoutée dans les produits (notamment par le biais d'une innovation mineure ou majeure). L'introduction d'une marque « bas de gamme » (stratégie 3) tendrait également à disparaître, car elle mine les relations avec la distribution et exige de forts investissements dont la rentabilisation est plus incertaine que dans les autres options stratégiques évoquées. Enfin, de nouvelles stratégies émergent (stratégie 5), soit le rapprochement en termes d'image des MDD lorsque celles-ci occupent des positions dominantes ou encore le développement de produits « haut de gamme » où les MDD sont encore peu présentes. Ces stratégies ne sont pas exclusives et les gestionnaires jouent sur leurs gammes de produits pour développer des approches combinées (notamment les stratégies 2 et 4 ).

Les résultats de la recherche tels que présentés ont été obtenus par une démarche qualitative ne permettant pas une extrapolation ou une généralisation des conclusions à l'ensemble des marques nationales gérées au Québec. Ils indiquent toutefois une tendance qui permet d'éclairer la problématique des stratégies de marque face aux MDD au Québec.

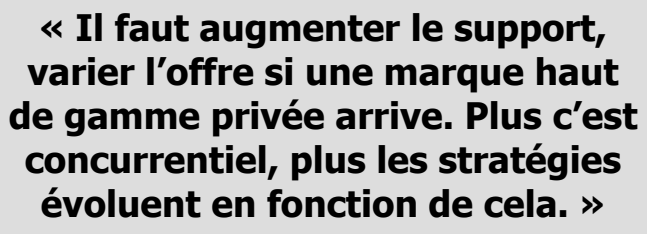

Ainsi, d'autres pistes de recherche sont envisageables pour l'avenir, notamment la confrontation des conclusions obtenues à la vision des consommateurs d'une part et des détaillants d'autre part sur d'autres catégories de produits que celles de l'industrie des aliments et boissons que nous avons étudiées.

La question des stratégies de marques face aux MDD reste pertinente et actuelle. Que feront en effet les fabricants si les MDD atteignent au Québec des parts de marché de plus de $40 \%$ en volume, comme cela est actuellement le cas en Suisse $(54,1 \%)$ ou en GrandeBretagne $(40,8 \%)$ depuis plus de trois années consécutives $^{12}$ ?

\section{Notes biographiques}

Catherine Parissier a obtenu son doctorat en Stratégie et management des organisations en 2003 à l'Université des Scien- 
ces et Technologies de Lille (France) et est actuellement professeure adjointe au département de marketing de la Faculté d'administration de l'Université de Sherbrooke. Depuis plus de 15 ans, Catherine Parissier enseigne en France et au Canada au niveau des programmes de premier, second et troisième cycle en recherche qualitative, marketing international et marketing relationnel. Ses intérêts de recherche sont surtout centrés sur le concept de valeur perçue par le consommateur et elle est membre de la Chaire Bombardier de gestion de la marque.

Julie Laurence a effectué un baccalauréat en administration des affaires, option marketing, à l'École des Hautes Études Commerciales de Montréal, suivi d'une maîtrise en administration avec un cheminement de type recherche, également dans le domaine du marketing, à l'Université de Sherbrooke. $\mathrm{Au}$ cours de ses études, ses intérêts de recherche se situaient principalement en gestion de la marque, plus spécifiquement dans le domaine agroalimentaire. Mme Laurence est présentement vice-présidente de AssurExperts Laurence, Vadnais et associés où elle entreprend la relève de ce cabinet en assurances de dommages.

\section{Notes et références}

1 Castonguay A. (2003). «Quand Loblaw achète au Québec », Commerce, vol. 104, $\mathrm{n}^{\circ} 11$, novembre, p. 37.

2 «MDD : toujours plus », Marketing Magazine (France), $\mathrm{n}^{\circ} 74$, novembre 2002 .

3 Kotler P., B. Dubois et D. Manceau (2003). Marketing Management, $11^{\mathrm{e}}$ édition, Paris, Pearson Éducation.
4 Vézina V. (2003). «L'année de l'incertitude », Affaires Plus, vol. 26, p. 36-39.

5 Lord V. (2003). « Le palmarès des marques maison, sondage exclusif Léger Marketing. La revanche des marques maison », Commerce, vol. 104, n 3, p. 29.

6 Notamment Hoch S.J. (1996). «How should National Brands Think about Private Label », Sloan Management Review, vol. 37, n 2, winter, p. 89-102; Verhoef, P.C., E.J. Nijssen et L.M. (2002). «Strategic Reactions of National Brands Manufacturers towards Private Labels : An Empirical Study in the Netherlands ", European Journal of Marketing, vol. 36, $\mathrm{n}^{\circ} 11 / 12$, p. 1309-1327.

7 Sayman S., S.J. Hoch et J.S. Raju (2002). « Positioning of Store Brands », Marketing Science, vol. 21, $\mathrm{n}^{\circ} 4$, automne, p. 378-398.

8 Ministère de l'Agriculture, des Pêcheries et de l'Alimentation du Québec (MAPAQ).

9 Exclusion faite de leurs magasins à escompte et dépanneurs.

10 Base de données des marques québécoises gérées au Canada élaborée par la Chaire Bombardier de Gestion de la marque (Faculté d'administration de l'Université de Sherbrooke) au cours de l'année 2004.

11 Les chiffres indiqués dans le tableau 1 sont exprimés en pourcentage de l'échantillon final (21 marques).

12 Rapport «Les marques de distributeurs » publié sur le site français de la Fédération des entreprises du commerce et de la distribution (mars 2005), www.fcd.asso.fr. 